International Journal of Artificial Intelligence \& Applications (IJAIA) Vol. 6, No. 3, May 2015

\title{
OPTICAL FLOW BASED ANALYSIS OF LEUKOCYTE DYNAMICS IN MICROCIRCULATION
}

\author{
Mohamed Maher Ben Ismail, Ouiem Bchir and Abdelmonim Artoli \\ College of Computer and Information Sciences, CS dept., King Saud University, Riyadh, \\ KSA
}

\begin{abstract}
Tracking of leukocytes in vivo and recording their dynamics with intravital microscopy is an effective technique for further understanding of the mechanism of inflammation and the influence of drug delivery in microcirculation. Though the technique is well established, automated quantitative analysis of the captured video frames is lacking in the literature. In this paper we adopt a duality based TV-L1 optical flow to analyze leukocyte behavior using in vivo video microscopy. Typically, we exploit the ability of this approach to conserve discontinuities in the flow field through the regularization of the Total Variation $(T V)$. On the other hand, the L1 norm overcomes the sensitivity to outliers. In the proposed framework, we submit the input images to a structure-texture decomposition in order to overcome the illumination changes causing violations in the optical flow constraint. Moreover, to further reduce the sensitivity to sampling artifacts in the image data, we apply a median filter into the numerical scheme. Using this technique, it is possible to directly compute the trajectories of leukocytes and their physical interaction parameters directly from the video frames. We have compared the obtained hydrodynamic fields with those obtained via simulation and have found that duality based TV-L1 optical flow approach is computationally less costly and reveals promising results.
\end{abstract}

\section{KEYWORDS}

Mechanism of inflammation, leukocyte dynamics, Optical flow.

\section{INTRODUCTION}

Since the 1800s, intravital microscopy has yielded direct visual observation of living circulatory beds. Thus, it has boosted the research related to inflammatory processes. This invention serves to analyse and investigate the dynamic behaviour of leukocytes. Investigating the leukocytes flow and their interaction with the endothelial wall in venules is one of the most important stages to understand leukocyte recruitment and analyse their response to external stimulation. Using these techniques and simple analysis, it is difficult to determine the parameters influencing the process and the hydrodynamic interaction among the complex blood components within the small venule regions. Properties such as the velocity, the acceleration and the flux are difficult to obtain within acceptable accuracy [1, 2]. More specifically, the analysis and investigation of leukocyte activation, adhesion, arrest, recruitment and subsequent rolling are perceived as the key answers for several challenges faced by the immunology researchers. In other words, understanding such processes would effectively contribute in the development of anti-inflammatory drugs such as modulators and blockers. In fact, the recruitment process is usually observed in post venules in the microcirculation. The main assumption here is that the adhesion cascade and the corresponding rolling process are mediated by a number of chemoattractants on the endothelial cell surface, selectins, integrins and other mediators on the surface of the leukocytes, and in the tissue. This mechanism is triggered by margination of free flowing leukocytes toward the endothelial wall. The interaction of leukocytes with surrounding erythrocytes yields deformation and pushing of the leukocytes to the wall $[3,4]$. Selectin mediators, such as E-Selectin, P-selectin 
and L-selectin, or their ligands assist the rolling captured leukocyte. Leukocytes which the rolling speed is decelerated by the CD18 integrins adhere to the endothelium with the mediation of Eselectins. Leukocytes change shape and transmigrate through the endothelium because of the existence of exogenous chemo attractants. The process aims at isolating and/or eradicating the irritants along with repairing the inflamed tissue. The process ends when the leukocyte is extravasated [5]. Despite the reported efforts to run and investigate in vivo and in vitro experiments, along with numerical simulations of leukocytes adhesion and recruitment, the phenomena has not given away all his secrets. Intensive investigation are still launched to determine the importance of the mechanism for different pathogenic and immunological activities such as kidney failure [6], cancer drug delivery and allergies [6, 7]. Moreover, the precise dynamical mechanisms by which molecular mediators facilitate leukocyte arrest are still undefined. Researchers have conducted intensive experiments in order to understand the complexity of the problem $[9,10,56]$. Leukocytes rolling has been investigated through several numerical simulations [11, 12]. The authors proposed a numerical simulation aiming at (i) obtaining detailed patterns for velocities and wall shear stress in a four dimensional nonNewtonian shear thinning model, (ii) identifying the role of localized shear stress profiles on the surface of leukocytes, and (iii) investigating the influence of clustering leukocytes on the endothelial wall shear stress. However, most of these assumptions have not been confirmed using real data. The main objectives of this paper is to use computer vision techniques to confirm the assumptions and conclusions in [12]. Moreover, the earliest efforts to understand leukocyte behaviours were constrained by the subjective manual analysis of the analyst rely on the subjective interpretation of the expert. Our aim is to propose a computer vision framework to support the analysis and the understanding of leukocyte trafficking and their interaction with microvessel walls based on a sequence of images. Relying only on optical flow technique, we seek a possibility for accurately determine governing hydrodynamic parameters and flow fields from a given set of itravital video frames of clusters of leukocytes moving through a rat venule of 20 micrometers in diameter. Instantaneous velocities and accelerations need to be determined in order to track each leukocyte and compute the net force acting on it. Since the captured video frames are two dimensional, we have neglected the influence of the third dimension. Each leukocyte has its own dimensions and will be treated as an individual object interacting with the surrounding. The influence of each object on its neighbors will completely be computed from analyzing the changes in successive frames as captured by image analysis using the optical flow method.

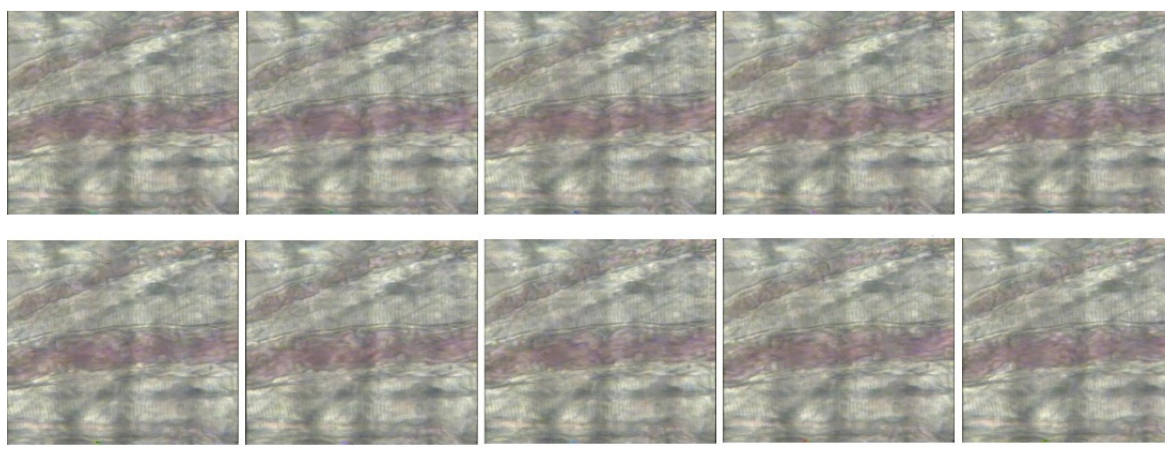

Figure 1: Ten successive typical frames of rolling and adherent leukocytes from in vivo color video clip

Figure 1 shows typical frames of rolling and adherent leukocytes from in vivo color video clip. As one can notice, adherent and moving leukocytes correspond to high intensity spheres compared with their neighbouring pixels. A natural solution to exploit this fact would be the use of an intensity threshold based technique to detect leukocytes in each frame. However, methods relying on a single threshold are not able to detect the "blurred" leukocytes as shown in Figure 1. 
Moreover, the discrimination between moving and adherent leukocytes is usually investigated in inflammation reaction experiences. Spatio-temporal feature extracted from successive frames has proven to be a good alternative to detect moving and/or adherent leukocytes. Thus, the proposed system integrates a version of the duality based TV-L1 optical flow algorithm in [40]. It exploits the ability of this algorithm to conserve discontinuities in the flow field through the regularization of the Total Variation (TV). On the other hand, the integrated L1 norm overcomes the sensitivity to outliers. In the proposed framework, we submit the input images to a structure-texture decomposition to overcome the illumination changes causing violations in the optical flow constraint. Moreover, to further reduce the sensitivity to sampling artefacts in the image data, we apply a median filter into the numerical scheme. This system can be used as an automated leukocyte detection algorithm within the microvasculature using video microscopy in vivo. The remainder of this paper is organized as follows. In section 2, we highlight related leukocyte recognition/detection techniques. In section 3, we present the optical flow estimation algorithm we adopt to investigate the dynamic behaviour of leukocytes. The experiments conducted to assess the proposed approach are outlined in section 4. Finally, section 5 contains the conclusions.

\section{RELATED WORKS}

Identification of leukocytes within intravital microscopy emerged as an active research field in various laboratories conducting inflammation related experiments. More specifically, the adhesion of rolling leukocytes to the vascular endothelium represents a key process of inflammation, and plays a fundamental role in our immune system [41]. For instance, continuous formation and breakage of bonds between the ligands on the endothelium and the corresponding selectin adhesion molecules on the leukocyte surface yield slower rolling leukocyte [42]. Various parameters related to rolling leukocytes, such as the number of rolling cells passing per unit time (flux of rolling leukocyte), the rolling leukocyte volume fraction and the rolling leukocytes per unit length are usually considered in order to design appropriate anti-inflammatory drugs [42,39]. However, the successful estimation of these parameters has been affected by the manual procedures performed by the laboratory technicians who may have to visually detect and analyze thousands of leukocytes in one video sequence. Recently, in order to assist technicians and overcome their potential distraction when investigating hundreds of frames, automated leukocytes identification systems has been proposed [50]. These systems have inherited various automatic object detection approaches [43]-[49]. In [51], various object recognition approaches were assessed. One of these approaches relies on convolution based filtering [53], feature-based classifiers [53, 54], and contour-based approaches represent alternative approaches [54]. One of the most popular Contour-based approaches is the Hough transform (HT) [43] which relies on a parametric representation in the parameter space in order to detect edge pixels vote for a shape. However, HT is mainly constrained by the identification accuracy of peaks in the parameter space. In fact, arc segments and spurious line yield wrong peaks. Notice that improved HT algorithms aiming at solving this issue have proved to be computationally expensive [45]. Another famous object detection approach consists in the Edge Radius Symmetry (ERS) transform which has been proposed to identify bronchi in CT lungs images [45]. Also, mathematical morphology has served as foundation of several object detection techniques [46]. In [47], the authors outlined a framework to detect leukocytes in contact with the vessel wall. Their approach relies on neural network that is trained with synthetic images generated using stochastic model. Another technique relying on image layer sets has been proposed in [48]. Cells detection is based on a search within the sets of image layers. A low-contrast object detection is achieved in [49] using the optical flow [21] which integrates the temporal information. This approach assumes that the width of the transition of image edge should be larger than the width of the perframe motion shift. Estimating the motion between video frames, referred to as optical flow estimation (OFE) finds application in various research domains such as image registration, video compression, motion detection, and video summarization. Researchers coupled optical flow with several techniques using frame sequences extracted from raw videos. Namely, some of the most 
used techniques are gradient based method, region based method, energy based method, Bayesian and phase based methods[16, 17]. Also, most of these optical flow based techniques require a large number of operations in order to reasonable performance. Moreover, more dense and accurate measurements of the optical flow velocity are required by some applications. The usage of high frame-rate sequences has enhanced the estimation accuracy of these techniques. Also, this yields less motion aliasing. Sequences capture at a high frame rate overcomes large velocity challenge, and complex images with high spatial bandwidths and low velocities. In [18], a method for block-based motion vector estimation at standard frame rate using high frame-rate video sequence is proposed. The authors outlined an iterative block matching framework using a high frame-rate sequence in order to produce motion vectors at 30 frames per second. In [19], the authors proposed an accurate estimation of the optical flow from high frame-rate sequence. The authors assume spatial smoothness and brightness constancy of the scene. On the other hand, its quadratic formulation makes it sensitive to outliers. In [22], the authors tackled this issue through a robust formulation of the problem. Other researchers investigated various robust functions [23]. These spatially-discrete formulations are perceived as "baseline" optical flow formulation approaches. Other significant non-classical approaches adopt image segmentation [27, 28], oriented smoothness $[24,25]$ or rigidity constraints $[25,26]$. Actual technique to overcome large motions is coarse-to-fine estimation [29, 30]. Similarly, high-order filter constancy [31] and/or texture decomposition [26] yield the reduction of lighting changes influence. Also, minimizing non-convex energies [24] is achieved using bicubic interpolation-based warping [32], temporal averaging of image derivatives [32 and graduated non-convexity [33]. On the other hand, median filtering after each incremental estimation step serves to discard outliers [32]. Median filtering heuristic enhances the robustness of methods sensitive to outliers, and improves the accuracy of all methods tested in [34]. Also, least median squares estimation has been used to discard outliers in flow estimation [35]. Similarly to median filtering, bilateral filtering changes the original energy function. Notice that model formulation which involves the L1 robust penalty is usually associated with specialized total variation (TV) optimization [36].

In this work, we adopt a version of the duality based TV-L1 optical flow algorithm in [40]. We exploit the ability of this approach to conserve discontinuities in the flow field through the regularization of the Total Variation (TV). On the other hand, the L1 norm overcomes the sensitivity to outliers by applying. In the proposed framework, we submit the input images to a structure-texture decomposition in order to overcome the illumination changes causing violations in the optical flow constraint. Moreover, to further reduce the sensitivity to sampling artifacts in the image data, we apply a median filter into the numerical scheme.

\section{OPTICAL FLOW MODEL}

Let $I_{1}$ and $I_{2}:\left(\Omega \subseteq R^{2}\right) \rightarrow R$ be two image frames. The optical flow can be formulated as the minimization of an image-based error criterion together with a regularization force in order to find the disparity map u $: \Omega \rightarrow R^{2}$ between the two images. In this work, the image similarity score is measured with respect to the intensity difference between pixels. Hence, the target disparity map $\mathrm{u}$ is the minimizer of

$$
\int_{\Omega}\left\{\lambda \phi\left(I_{1}(\mathrm{x})-I_{2}(\mathrm{x}+\mathrm{u}(\mathrm{x}))\right)+\psi(u, \nabla u, \ldots)\right\} d x,(1)
$$

where the term $\phi\left(I_{1}(\mathrm{x})-I_{2}(\mathrm{x}+\mathrm{u}(\mathrm{x}))\right)$ represents the image data typicality, and $\psi(u, \nabla u, \ldots)$ is the regularization term. The parameter $\lambda$ is the weighing factor of the two terms. Selecting $\varphi(x)=x^{2}$ and $\psi(\nabla u)=|\nabla u|^{2}$ yields to Horn-Schunck model [21]. On the other hand, setting $\varphi(x)$ to $x$ and $\psi(\nabla u)$ to $|\nabla u|$ results in the following energy.

$$
E=\int_{\Omega}\left\{\lambda\left|I_{1}(\mathrm{x})-I_{2}(\mathrm{x}+\mathrm{u}(\mathrm{x}))\right|+|\nabla u|\right\} d x,(2)
$$

As it can be seen, $E$ consists of a total variation regularization and an L1 data penalty term. Moreover, the regularization term and the data term are not continuously differentiable. 
Therefore, in order to apply numerical optimization technique, we replace $\varphi(x)$ and $\psi(\nabla u)$ with differentiable approximations, $\varphi_{\varepsilon}\left(\mathrm{x}^{2}\right)=\sqrt{\mathrm{x}^{2}+\varepsilon^{2}}$ and $\psi(\nabla u)=\sqrt{|\nabla u|^{2}+\varepsilon^{2}}$, respectively.

Based on two images $I_{1}$ and $I_{2}$, let $u$ be an $N$-dimensional displacement map. The first order image residual $\rho\left(u, u_{1}, x\right)$ with respect to a given disparity map $u_{1}$ is $I_{2}\left(\mathrm{x}+\mathrm{u}_{1}\right)+\left(\nabla I_{2}, u-u_{1}\right)-I_{1}(\mathrm{x})$. On the other hand, the energy can be formulated as follows:

$$
E_{\theta}=\int_{\Omega}\left\{\sum_{d}\left|\nabla u_{d}\right|+\sum_{d} \frac{1}{2 \theta}(u(\mathrm{~d})-\mathrm{v}(\mathrm{d}))^{2}+\lambda\left|\rho\left(\mathrm{v}, \mathrm{u}_{1}, \mathrm{x}\right)\right|\right\} d x,(3)
$$

where $u_{d=\{1, \ldots, \mathrm{N}\}}$ is the $d^{\text {th }}$ component of $u$, and $\theta$ is a constant such that $v$ is a close approximation of $u$.

Alternating optimization steps, with respect to $u$ and $v$, of this convex problem yield the following solutions [32]:

$$
v=u+\left\{\begin{array}{cl}
\lambda \theta \nabla I_{2} & \text { if } \mid \rho\left(u, u_{1},\left.x|<-\lambda \theta| \nabla I_{2}\right|^{2}\right. \\
-\lambda \theta \nabla I_{2} & \text { if } \mid \rho\left(u, u_{1},\left.x|>\lambda \theta| \nabla I_{2}\right|^{2}\right. \\
-\rho(\mathrm{u}) \frac{\nabla I_{2}}{\left|\nabla I_{2}\right|^{2}} & \text { if } \mid \rho\left(u, u_{1},\left.x|\leq \lambda \theta| \nabla I_{2}\right|^{2}\right.
\end{array}\right.
$$

On the other hand, the structure-texture decomposition aiming at reducing the sensitivity to illumination changes is performed using the robust model in [56]. This model relies on Total Variation (TV) approach, and the structural part of a value image $I(x)$ is formulated as the solution of the following problem

$$
\min _{I_{s}} \int_{\Omega}\left\{\left|\nabla I_{s}\right|+\frac{1}{2 \theta}\left(I_{s}-I\right)^{2}\right\} d x,(5)
$$

Then, we estimate the textural part $I_{T}(\mathrm{x})$ as the difference between the denoised image and the original one. This procedure overcomes the problem of artifacts caused by shading reflections and shadow in the images.

We use the standard incremental multi-resolution technique in [27] to minimize the energy in (3), and estimate the flow fields corresponding to large displacements. This approach starts by estimating the optical flow at a coarse layer. Then, the obtained flow is used to warp the second image toward the first at the next finer layer. Finally, the flow increment is estimated based on these two images. Notice that each layer is recursively downsampled from its neighbour lower layer. The number of pyramid layers is defined in a way the highest layer has an average width of 25 pixels. At each layer, as suggested in [27], 10 warping steps are performed in order to estimate the flow increment. Also, the warping step requires the linearization of the data term. On the other hand, the pyramid prolongation and restriction for image intensities, flow vectors, and the dual variable are different. In fact, gray values can simply be averaged. Notice that flow vectors require scaling factor between the pyramid levels in order to induct valid displacement vectors on each pyramid layer. In this work, we adopt image pyramids with a factor of 2 down-sampling. The restriction operator is designed as a combination of a low pass $5 \times 5$ binomial filter and subsequent down-sampling. As suggested in [32], we adopt bi-cubic look-up to estimate the intensity value $I_{2}\left(x+u_{1}\right)$ and the derivatives of $I_{1}$ which are calculated using the five-point stencil. Finally, If $u_{1}$ is a good approximation for $\mathrm{u}$, the optical flow constraint states that $I_{1}(\mathrm{x}) \approx I_{2}\left(x+u_{1}\right)$ - Considering this fact when estimating image derivatives implies that $\nabla I_{1}(x)$ is a good approximation for $\nabla I_{2}\left(x+u_{1}\right)$. 


\section{EXPERIMENTS}

The video frames used in this work were provided by Artoli et al. from a previous work [12] to which we refer the reader for more information on the experiment, ethical clearance and data collection. The obtained video frames are for leukocytes moving through a venule in an inflamed region of a Wistar male rat. We have conducted a number of optical flow analysis on consecutive video frames of moving leukocytes. For each two consecutive frames $I_{1}$ and $I_{2}$, the velocity was captured pixel wise as outlined in Section 3.

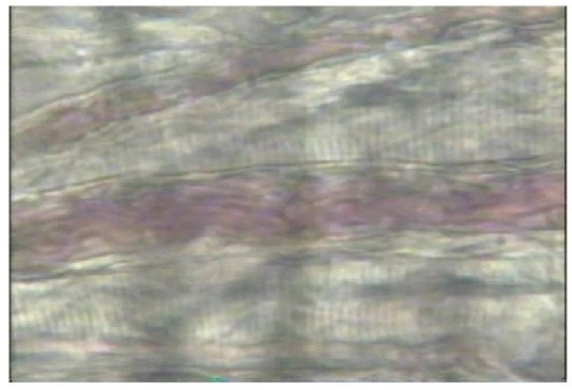

(a)

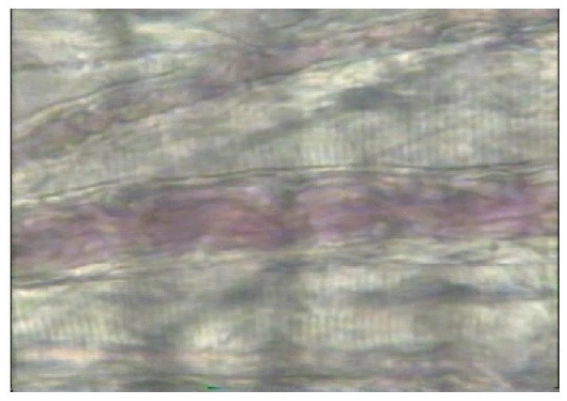

(b)

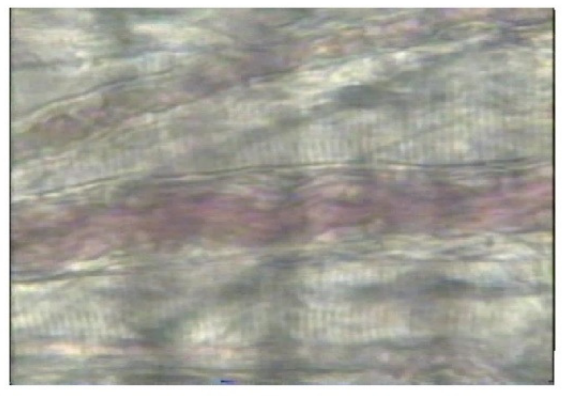

(d)

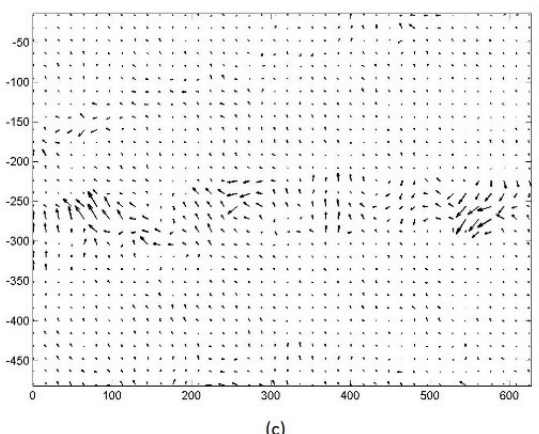

(c)

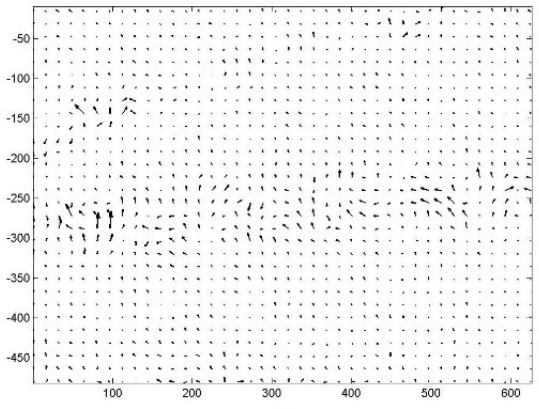

(e)

Figure 2: Three successive sample frames (left), and their corresponding optical flow velocity profiles.

This is actually the velocity of moving blood components. Sample velocity profile for the two consecutive frames $I_{i}$ and $I_{i+1}$ shown in Figure 2.(a) and Figure 2.(b), respectively, is given in Figure 2.(c). Similarly, the velocity profile of the two next frames $I_{i+1}$ and $I_{i+2}$ is shown in Figure 2.(e). Figure 2.(c) proves that large velocity magnitudes and more swirling appear close to the endothelial walls, showing the initiation of the rolling process for the leukocytes. This may be explained as follows. The leukocytes move with lower velocities than the main stream of the blood components. When they are close to the endothelial wall, the flow between the leukocyte and the endotheial wall moves faster and rotates more around the slowly moving spherical leukocyte. 
Sample velocity profiles and their corresponding acceleration, estimated as the first order differential of the velocity vectors of moving blood components, are depicted in Figure 3. As one can see, the acceleration map in Figure 3.(c) corresponds to the two velocity profiles in Figure 3.(a) and Figure 3.(b). Similarly, Figure 3.(f) illustrates the acceleration of the the two velocity profiles in Figure 3.(d) and Figure 3.(g). From these Figures we realize that the characteristics of the flow fields, obtained using optical flow, is more robust and time efficient than conducting regular image segmentation on the moving components. Moreover, these results reveal similar flow characteristics to those reported in [18]. For example, the swirling nature of the flow and the traping stagnant regions have been captured. The 3D nature of the flow may be noticed from the red velocity regions (see Figure 3.(b)-(e)-(h)) where higher disturbance occurs due to the rotation of the leukocytes and their motion toward the endothelial wall which pushes the flow in a smaller tunnel and increases their velocity. Figure 4 compares between simulation and optical flow based analysis results of the velocity fields. The artwork accompanying this paper elaborates on this similarity.
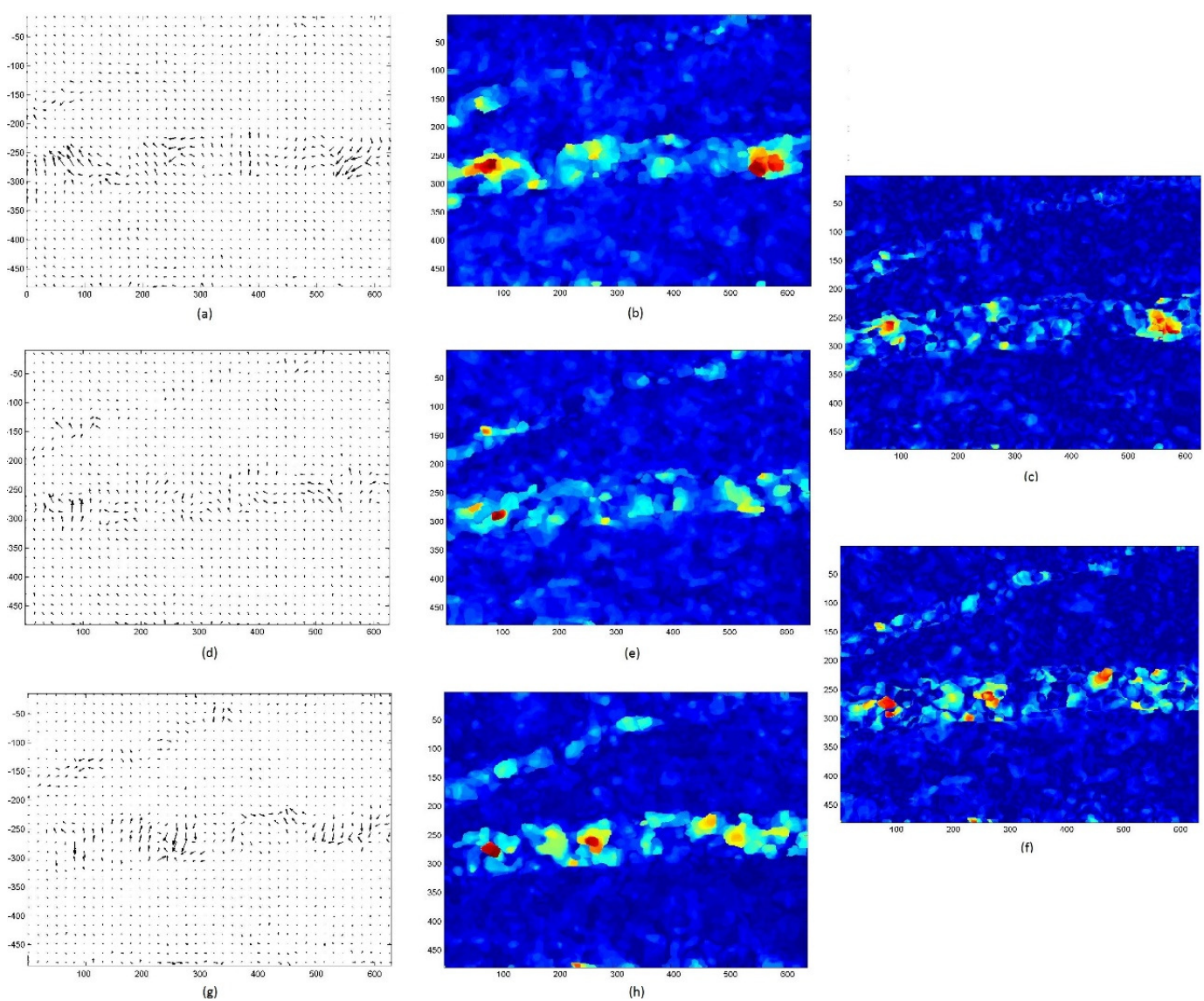

Figure 3: Sample velocity profiles (left and center) and their corresponding acceleration map (right).

The maximum velocity reported in the literature was $6-12 \mu \mathrm{m} / \mathrm{s}$ for the leuckocytes [18]. In this work, we report a velocity range of $3.7-11.6 \mu \mathrm{m} / \mathrm{s}$. The average velocity of leukocytes (the blue objects in Figure 3(b), Figue 3.(e), and Figue 3.(h)) is approximately $5.4 \pm 1.3 \mu \mathrm{m} / \mathrm{s}$. On the other hand, the acceleration range is $0-0.99 \mu \mathrm{m} / \mathrm{s}^{2}$, and the average acceleration is approximately $0.019 \pm 0.02 \mu \mathrm{m} / \mathrm{s}^{2}$. 
International Journal of Artificial Intelligence \& Applications (IJAIA) Vol. 6, No. 3, May 2015

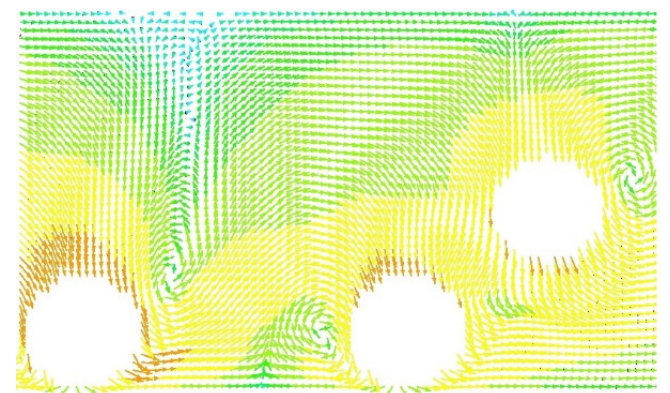

(a)

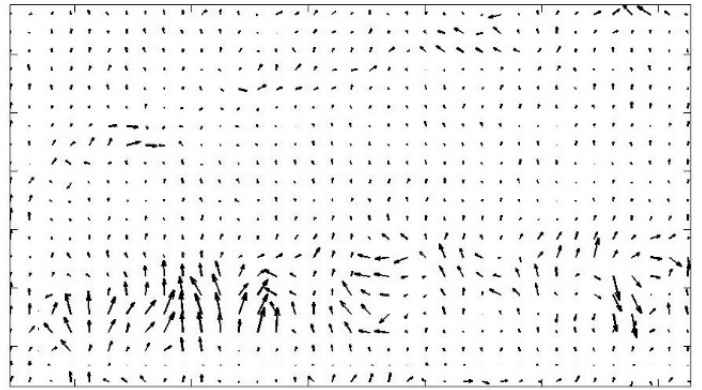

(b)

Figure 4: Comparison between (a) simulated velocity field [12], and (b) optical flow based velocity field.

\section{CONCLUSIONS}

Since it was introduced by Gibson in the 1940s, optical flow techniques have been used in many applications to estimate the relative motion of successively recorded events in dynamic environments. In this work we have demonstrated that this technique yields reasonably accurate results when used in tracking leukocyte dynamics in microcirculation. The range of obtained velocity and acceleration is comparable to that reported in the literature. As the shear stress and the vorticity play important roles in biomechanics, we have noticed that optical flow is very sensitive to discontinuity in depth and brightness which suggest that it will yield good estimates for the stress and the three dimensional nature of hydrodynamic phenomena in discovering regions of complex fluid flow. However, further benchmarking to compute the accuracy of the method is a subject for future work.

\section{REFERENCES}

[1] G. W. Schmid-Schonbein, S. Usami, R. Skalak, and S. Chien, "The interaction of leukocytes and erythrocytes in capillary and postcapillary vessels," Microvasc. Res., vol. 19, pp. 45-70, 1980.

[2] U. H. von Andrian, J. D. Chambers, L. M. Mcevoy, R. F. Bargatze, K. E. Arfors, and E. C. Butcher, "Two-step model of leukocyte-endothelial cell interaction in inflammation: Distinct roles for lecam-1 and the leukocyte beta 2 integrins in vivo", Proc Natl Acad Sci, vol. 88, no. 17, pp. 7538-42, U S A. 1991.

[3] O.K. Baskurt, R.A. Farely, H.J. Meiselman, "Erythrocyte aggregation tendency and cellular properties in horse, human, and rat: A comparative study", American Journal of Physiology-Hearth and Circulatory Physiology, vol. 273, pp.2604-2612, 1996.

[4] R.M. Rao, L. Yang, G. Garcia-Cardena, F.W. Luscinskas, "Endothelial-dependent mechanisms of leukocyte recruitment to the vascular wall", Circulation Research vol. 101, pp. 234-247, 2007.

[5] A. Evans, D.A. Calderwood, "Forces and bond dynamics in cell adhesion", Science 316 (5828), pp. 1148-1153, 2007.

[6] K. Sigbartl, K. Ley, "Leukocyte recruitment and acute renal failure", Journal of Molecular Medicine, vol. 82, pp.1432-1440, 2004.

[7] R. Lever, C.R. Page, "Novel drug development opportunities for heparin", Nature Review Drug Discovery 1 (2), pp. 140-148, 2002.

[8] Z. Szekanecz, A.E. Koch, "Therapeutic inhibition of leukocyte recruitment in inflammatory diseases", Current Opinion in Pharmacology, 4 (4). pp. 423, 2004.

[9] Schmid-Scho“ nbein, G.W., Barroso-Aranda, J., Chavez, R., "Microvascular leukocyte kinetics in the flow state. In: Boccalon", H. (Ed.), Vascular Medicine. pp. 349-352, Elsevier, Amsterdam, 1993.

[10] Krasik, E.F., Yee, K.L., Hammer, D.A., “Adhesive dynamics simulation of neutrophil arrest with deterministic activation”. Biophysical Journal 91 (4), pp. 1145-1155, 2006.

[11] Munn, L.L., Melder, R.J., Jain, R.K., "Role of erythrocytes in leukocyte-endothelial interactions: mathematical model and experimental validation". Biophysical Journal, 71 (1), pp. 466-478. 1996.

[12] A.M. Artoli,, A. Sequeiraa, A.S. Silva-Herdadeb, C. Saldanhab, "Leukocytes rolling and recruitment by endothelial cells: Hemorheological experiments and numerical simulations", Journal of Biomechanics 40, pp. 3493-3502, 2007. 
International Journal of Artificial Intelligence \& Applications (IJAIA) Vol. 6, No. 3, May 2015

[13] Rosa' rio, H.S., Saldanha, C., Martins e Silva, J., "The effect of velnacrine in lipopolysaccharideinduced leukocyte-endothelial interaction”. 21st European Conference on Microcirculation. Monduzzi Editore, Italy, 2000.

[14] Andonegui, G., Goyert, S.M., Kubes, P., "Lipopolysaccharideinduced leukocyte-endothelial cell interactions: a role for CD14 versus toll-like receptor 4 within microvessels". Journal of Immunology 169, pp. 2111-2119, 2002.

[15] Silva, A.S., Saldanha, C., Martins, E., Silva, J., "Effects of velnacrine maleate in the leukocyteendothelial cell interactions in rat cremaster microcirculatory network". Clinical Hemorheology and Microcirculation 36 (3), pp. 235-246, 2007.

[16] J. L. Barron, D. J. Fleet, and S. S. Beauchemin, "Performance of optical flow techniques," Int. J. Comput. Vis., vol. 12, no. 1, pp. 43-77, 1994.

[17] C. Stiller and J. Konrad, "Estimating motion in image sequences," IEEE Signal Process. Mag., vol. 16, no. 7, pp. 70-91, 1999.

[18] D. Handoko, S. Kawahito, Y. Takokoro, M. Kumahara, and A. Matsuzawa, "A CMOS image sensor for focal-plane low-power motion vector estimation," in Proc. Symp. VLSI Circuits, pp. 28-29, 2000.

[19] S. H. Lim and A. El Gamal, "Optical flow estimation using high frame rate sequences," in Proc. Int. Conf. Image Processing, vol. 2, pp. 925-928, 2001.

[20] M. Ozuysal, P. Fua, and V. Lepetit, "Fast keypoint recognition in ten lines of code," in CVPR, 2007, pp. $1-8$.

[21] B. Horn and B. Schunck. "Determining optical flow", Artificial Intelligence, 16, pp. 185-203, 1981.

[22] M. J. Black and P. Anandan. "The robust estimation of multiple motions: Parametric and piecewisesmooth flow fields". CVIU, 63, pp. 75- 104, 1996.

[23] T. Brox, A. Bruhn, N. Papenberg, and J. Weickert. "High accuracy optical flow estimation based on a theory for warping". In ECCV, pages 25-36, 2004.

[24] H.-H. Nagel and W. Enkelmann. "An investigation of smoothness constraints for the estimation of displacement vector fields from image sequences". PAMI, vol. 8, no. 5, pp. 565-593, 1986.

[25] A. Wedel, T. Pock, and D. Cremers. "Structure- and motion-adaptive regularization for high accuracy optic flow". In ICCV, 2009.

[26] A. Wedel, T. Pock, J. Braun, U. Franke, and D. Cremers. "Duality TV-L1 flow with fundamental matrix prior". In IVCNZ, 2008.

[27] M. Black and A. Jepson. "Estimating optical-flow in segmented images using variable-order parametric models with local deformations". PAMI, vol. 18, no. 10, pp. 972-986, 1996.

[28] C. Lei and Y.-H. Yang. "Optical flow estimation on coarse-to-fine region-trees using discrete optimization”. In ICCV, 2009.

[29] J. Bergen, P. Anandan, K. Hanna, and R. Hingorani. "Hierarchical model-based motion estimation". In ECCV, pp. 237-252, 1992.

[30] A. Bruhn, J. Weickert, and C. Schn“orr. Lucas/Kanade meets Horn/Schunck, "combining local and global optic flow methods". IJCV, vol. 61, no. 3, pp. 211-231, 2005.

[31] F. Glaer, G. Reynolds, and P. Anandan. "Scene matching by hierarchical correlation". In CVPR, pp. 432-441, 1983.

[32] A. Wedel, T. Pock, C. Zach, D. Cremers, and H. Bischof. “An improved algorithm for TV-L1 optical flow". In Dagstuhl Motion Workshop, 2008.

[33] A. Blake and A. Zisserman. "Visual Reconstruction". The MIT Press, Cambridge, Massachusetts, 1987.

[34] Deqing Sun; Roth, S. ; Black, M.J., "Secrets of optical flow estimation and their principles", IEEE Conference on Computer Vision and Pattern Recognition (CVPR), pp. 2432 - 2439, 2010.

[35] A. Bab-Hadiashar and D. Suter. "Robust optic flow computation". IJCV, vol. 29, no. 1, pp. 59-77, 1998.

[36] C. Zach, T. Pock, and H. Bischof. "A duality based approach for realtime TV-L1 optical flow”. In DAGM, 2007.

[37] B. Horn. "Robot Vision". MIT Press, 1986.

[38] Alice Chan, Boris P. Kovatchev, Stacey M. Anderson, Marc D. Breton, "Systematic method to assess microvascular recruitment using contrast-enhanced ultrasound Application to insulin-induced capillary recruitment in subjects with T1DM", Computer Methods and Programs in Biomedicine, Vol. 102, no. 2, pp. 219-226, 2011.

[39] C. Zach, T. Pock, and H. Bischof. A Duality Based Approach for Realtime TV-L1 Optical Flow. Pattern Recognition (Proc. DAGM), pp. 214-223, 2007.

[40] J. Alper, "Searching for medicine's sweet spot," Science, vol. 291, pp. 2338-2343, 2001. 
International Journal of Artificial Intelligence \& Applications (IJAIA) Vol. 6, No. 3, May 2015

[41] M. G. oude Egbrink, G. J. Tangelder, D. W. Slaaf, and R. S. Reneman, "Influence of platelet-vessel wall interaction on leukocyte rolling in vivo," Circ. Res., vol. 70, pp. 355-363, 1992.

[42] K. Ley, "Leukocyte recruitment as seen by intravital microscopy," in Physiology of Inflammation. New York: Oxford Univ. Press, pp. 303-337, 2001.

[43] H. K. Yuen, J. Illingworth, and J. Kittler, "Detecting partially occluded ellipses using the Hough transform," Image Vis. Comput., vol. 7, pp. 31-37, 1989.

[44] J. Illingworth and J. Kittle, “A survey of the Hough transform,” CVGIP, vol. 44, pp. 87-116, 1988.

[45] F. Chabat, X. Hu, D. M. Hansell, and G. Yang, "ERS transform for the automated detection of bronchial abnormalities on CT of the lungs," IEEE Trans. Med. Imag., vol. 20, no. 9, pp. 942-952, 2001.

[46] S. Mukhopadhyay and B. Chanda, "Multiscale morphological segmentation of gray-scale images," IEEE Trans. Image Process., vol. 12, no. 5, pp. 533-549, 2003.

[47] M. Egmont-Peterson, U. Schreiner, S. C. Tromp, T. M. Lehmann, D.W. Slaaf, and T. Arts, "Detection of leukocytes in contact with the vessel wall from in vivo microscope recordings using a neural network," IEEE Trans. Biomed. Eng., vol. 47, no. 7, pp. 941-951, 2000.

[48] D. P. Mukherjee, N. Ray, and S. T. Acton, "Level set analysis for leukocyte detection and tracking," IEEE Trans. Image Process., vol. 13, no. 4, pp. 562-572, 2004.

[49] V. Markandey, A. Reid, and S. Wang, "Motion estimation for moving target detection," IEEE Trans. Aerosp. Electron. Syst., vol. 32, pp. 866-874, 1996.

[50] Gang Dong, Nilanjan Ray, Scott T. Acton: Intravital leukocyte detection using the gradient inverse coefficient of variation. IEEE Trans. Med. Imaging vol. 24, no. 7, pp. 910-924, 2005.

[51] M. Egmont-Petersen and T. Arts, "Recognition of radiopaque markers in X-ray images using a neural network as nonlinear filter," Pattern Recogn. Lett., vol. 20, no. 5, pp. 521-533, 1999.

[52] M. Egmont-Petersen and T. Arts, "Detection of implanted markers in radiographic image sequences," in Aachen Workshop on Medical Image Processing, T. Lehmann and K. Spitzer, Eds, , pp. 209-214, 1996.

[53] D. DeKruger and B. R. Hunt, "Image processing and neural networks for recognition of cartographic area features," Pattern Recognition, vol. 27, no. 4, pp. 461-483, 1994.

[54] M. Egmont-Petersen and E. Pelikan, "Detection of bone tumours in radiographs using neural networks," Pattern Anal. Applicat., vol. 2, no. 2, pp. 521-533, 1999.

[55] S. Lobregt and M. A. Viergever, "A discrete dynamic contour model," IEEE Trans. Med. Imag., vol. 14, pp. 12-24, 1995.

[56] L. I. Rudin, S. Osher, and E. Fatemi. Nonlinear total variation based noise removal algorithms. Physica D, 60:259-268, 1992.

[57] Dmitriy Shutin, Olga Zlobinskaya, "Application of information-theoretic measures to quantitative analysis of immunofluorescent microscope imaging", Computer Methods and Programs in Biomedicine, Vol. 97, no. 2, pp. 114-129, 2010.

\section{Authors}

Dr. Mohamed Maher Ben Ismail is assistant professor at the computer science department of the College of Computer and Information Sciences at King Saud University. He received his $\mathrm{PhD}$. degree in Computer Science from the University of Louisville in 2011. His research interests include Pattern Recognition, Machine Learning, Data Mining and Image Processing.

Dr. Ouiem Bchir is assistant professor at the computer science department, College of Computer and Information Sciences (CCIS), King Saud University, Riyadh, Saudi Arabia.She got her PhD from the University of Louisville, KY, USA. Her research interests are Spectral and kernel clustering, pattern recognition, hyperspectral image analysis, local distance measure learning, and Unsupervised and Semi-supervised machine learning techniques. She received the University of Louisville Dean's Citation, the University of Louisville CSE Doctoral Award, and the Tunisian presidential award for the electrical engineering diploma.

Prof. Abdel Monim Artoli is Professor of computational science, Computer Science Department, Vice-deanship for Development and Quality, Head of Alumni Unit, Department of Computer Science, College of Computer \& Information Sciences, King Saud University.
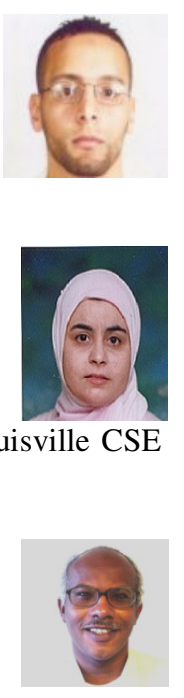